\title{
Synchronization of Synovial Chondromatosis and Mycobacterium intracellurae Infection in Olecranon Bursitis: A Case Report
}

\author{
Dong Hyun Kim, Seunggi Min, Hyun Joo Lee, Hee-June Kim, Hoseok Lee ${ }^{1}$, Jong Pil Yoon ${ }^{\square}$ \\ Departments of Orthopaedic Surgery and ${ }^{1}$ Radiology, Kyungpook National University School of Medicine, Daegu, Korea
}

\begin{abstract}
A 73-year-old woman presented with a recurrent cystic mass around her left olecranon. She had a history of 8 steroid injections due to elbow pain beginning 3 years ago and twice had undergone aspiration of olecranon bursitis that developed two months prior to presentation. She had been taking medications for hypertension and diabetes with no pertinent past history. On magnetic resonance imaging (MRI), there were multiple nodules in the olecranon bursa, which were isointense to muscle on T1-weighted images and hyperintense to muscle on T2-weighted images. Our initial diagnosis was synovial chondromatosis. On bursoscopy, masses of gray-white colored nodules were observed in the bursa. Finally, synovial chondromatosis and non-tuberculous mycobacterial infection were concurrently diagnosed. In conclusion, uncalcified synovial chondromatosis and rice bodies can have similar visual and MRI characteristics; therefore, we suggest that clinicians should be aware of the possibility of other infections in cases of this type.
\end{abstract}

(Clin Shoulder Ellbow 2019;22(1):46-49)

Key Words: Elbow; Synovitis; Synovial chondromatosis; Tuberculosis; Nontuberculous Mycobacterium

Non-tuberculous mycobacterial olecranon bursitis has already been described in several reports. ${ }^{1)}$ However, there are no reports describing secondary synovial chondromatosis in olecranon bursitis. As well, there are no reports indicating synchronization of non-tuberculous mycobacterial infection and synovial chondromatosis. Herein, we report a case of synovial chondromatosis associated with non-tuberculous mycobacterial infection in olecranon bursitis.

\section{Case Report}

A 73-year-old woman presented with a rapidly growing recurrent cystic mass around her left olecranon. She had a history of elbow pain and had received 8 steroid injections beginning approximately three years prior to presentation at our clinic. A cystic mass developed at the posterior aspect of the left elbow about two months prior to presentation. At a local clinic, the initial diagnosis was olecranon bursitis, and twice she had undergone aspiration for the effusion within the bursa. However, three weeks after the second aspiration, the cystic mass recurred and grew rapidly. As a result, she was referred to our clinic for further evaluation.

On physical examination, the mass size was approximately $20 \mathrm{~cm}$ long and $5 \mathrm{~cm}$ wide (Fig. 1). There was mild tenderness in the surrounding soft tissue, but no definite infection signs such as redness or warmth. The mass was soft and mobile. She had been taking medications for hypertension and diabetes, but there were no pertinent occurrences, such as a pulmonary tuberculous infection, in her past history. Plain radiography revealed soft tissue enlargement around the olecranon with an olecranon process spur but no evidence of arthritis, such as bone erosion, atrophy, or a destructive process (Fig. 2). Blood analysis indicated her erythrocyte sedimentation rate was $32 \mathrm{~mm} / \mathrm{hr}$ and her C-reactive protein level was normal at $0.1 \mathrm{mg} / \mathrm{dl}$.

Received July 9, 2018. Revised September 30, 2018. Accepted October 1, 2018.

Correspondence to: Jong Pil Yoon

Department of Orthopaedic Surgery, Kyungpook National University School of Medicine, 130 Dongdeok-ro, Jung-gu, Daegu 41944, Korea

Tel: +82-53-420-5628, Fax: +82-53-422-6605, E-mail: jpyoon@knu.ac.kr, ORCID: https://orcid.org/0000-0001-6446-6254

IRB approval: Kyungpook National University Hospital (No. KNUH 2018-06-030).

Financial support: This research was supported by the Basic Science Research Program through the National Research Foundation of Korea, funded by the Ministry of Science, ICT and Future Planning (2015R1C1A1A02036478). Conflict of interests: None. 


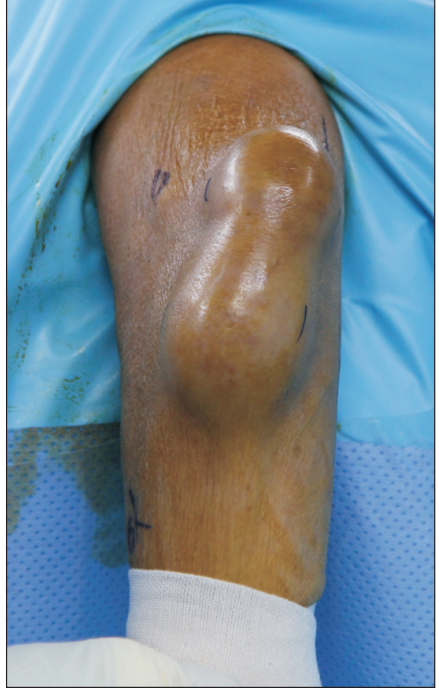

Fig. 1. A large mass (about $20 \mathrm{~cm}$ long and $5 \mathrm{~cm}$ wide) originating from the olecranon bursa on the posterior aspect of the left elbow was identified.

Although we initially considered recurrence of olecranon bursitis, because of a previous recurrence despite repeated aspirations over a two month period, ultrasonography was performed to determine whether other accompanying lesions were present. On ultrasonography, a mixed hypoechoic mass with a welldefined irregular shape was observed in the olecranon bursa. Subsequently, magnetic resonance imaging (MRI) was performed to discriminate the mixed hypoechoic mass.

On MRI, there were multiple nodules in the olecranon bursa, which were isointense to muscle on the T1-weighted images and hyperintense to muscle, though less intense than fluid, on the T2-weighted images. Following intravenous administration of gadolinium contrast, there was enhancement of the thin, smooth synovial membrane surrounding the olecranon bursa but no enhancement of the soft tissue nodules. The underlying bones appeared normal without joint involvement (Fig. 3). Based on the results, the radiologist suggested synovial chondromatosis as a preliminary diagnosis but noted pigmented villonodular synovitis and abscess as other possibilities.

We considered the presence of secondary synovial chondromatosis of chronic olecranon bursitis and planned for bursoscopic synovectomy and mass excision. We performed debridement of the inflammatory and proliferated synovial tissue around the olecranon bursa and found masses of numerous gray-white colored nodules in the bursa (Fig. 4). We considered several possibilities related to those results and performed a variety of relevant histological and microbiological confirmatory tests, including tissue biopsy and culture, and undertook realtime polymerase chain reaction (PCR) assays for mycobacterial tuberculosis and nontuberculous Mycobacterium (NTM).

Two weeks later, based on the histologic results, synovial chondromatosis was diagnosed. Subsequently, the non-
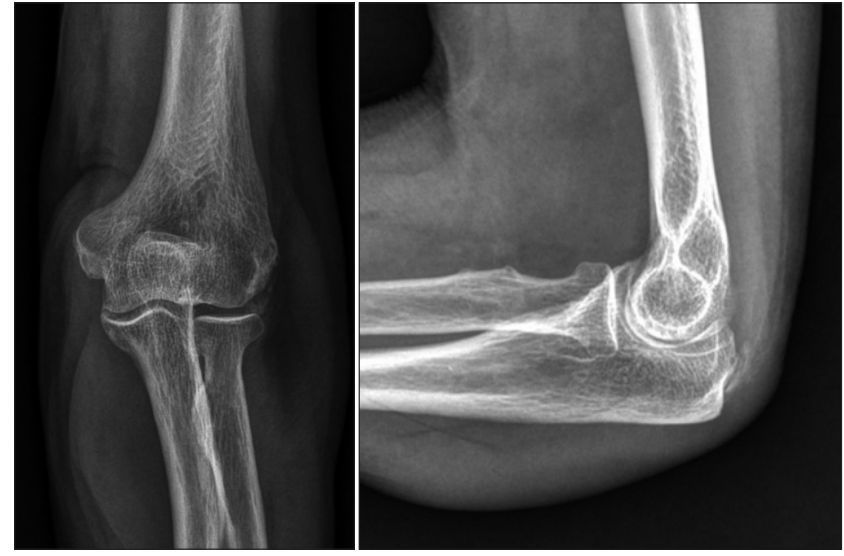

Fig. 2. Plain radiographs showing soft tissue enlargement around the olecranon with an olecranon process spur and calcification on triceps insertion but no evidence of arthritis, such as bone erosion, atrophy, or a destructive process.

tuberculous mycobacterial result was positive on real-time PCR. On those bases, empirical antimicrobial agents (clarithromycin, rifampin, and ethambutol) were administered to the patient for first 3 months regardless of in vitro susceptibility drug test. Three months after the operation, tissue cultures revealed the presence of Mycobacterium intracellulare. In vitro susceptibility testing of NTM species revealed sensitivity to clarithromycin, and the treatment drugs were changed to clarithromycin and ethambutol. At postoperative nine months, she had no symptoms or signs of recurrence and no medication-related complications.

\section{Discussion}

Synovial chondromatosis is a rare, mostly benign proliferation of the synovium of the joint, tendon, or bursa, and can result in the formation of loose bodies. The intra-articular form most commonly affects the knee joint. Extra-articular sites are uncommon, most often involving synovial sheaths or bursa of the hand or foot. ${ }^{2)}$ Some cases of synovial chondromatosis in elbow joints have been reported. ${ }^{3)}$ However, to our knowledge, there are no reports of synovial chondromatosis in olecranon bursitis. Matsumoto et al. ${ }^{4)}$ reported massive nonspecific olecranon bursitis with multiple rice bodies, but rice bodies are not the result of synovial chondromatosis but, rather, of non-tuberculous mycobacterial infection.

Non-tuberculous mycobacteria are present in the soil and water and can cause extra-pulmonary disease under immunocompromised conditions. Garrigues et al. ${ }^{1)}$ reported that $38 \%$ of previously reported patients with non-tuberculous mycobacterial olecranon bursitis take immunosuppressive drugs, such as systemic corticosteroids or immunomodulators, or they have an underlying disease such as diabetes mellitus. Several species of NTM have been associated with olecranon bursitis. The frequency of minor trauma to the elbow and the subcutaneous 

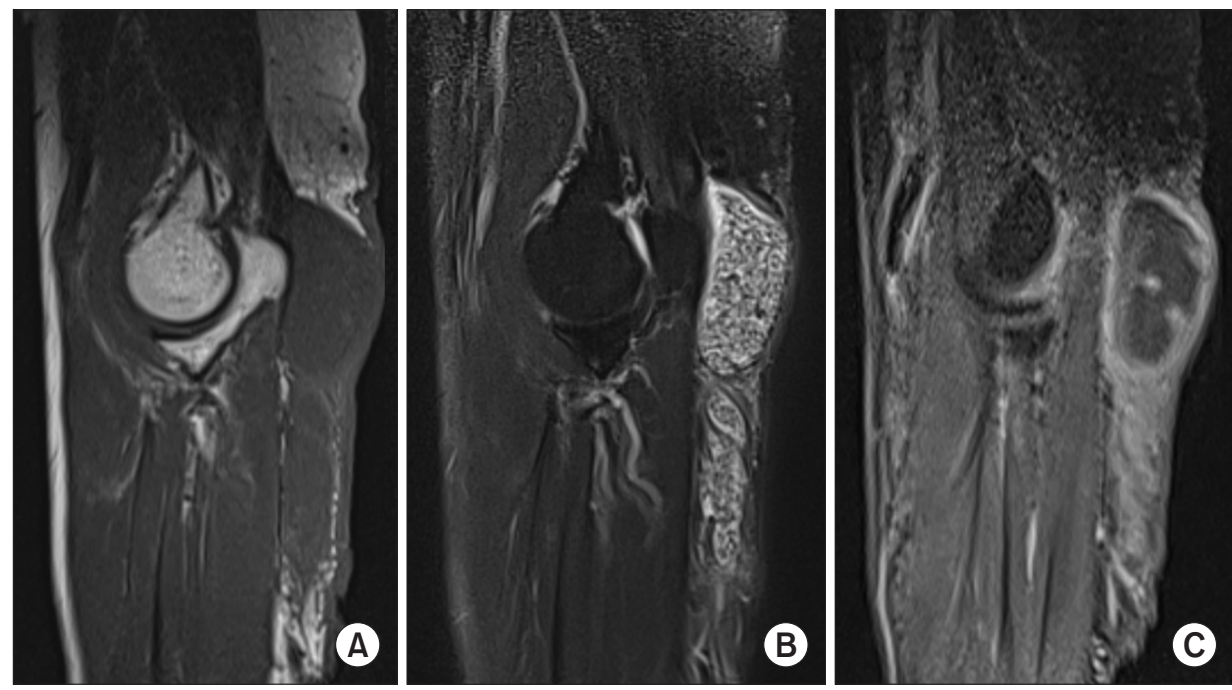

Fig. 3. (A) T1-weighted sagittal magnetic resonance imaging (MRI); There were multiple nodules inside the olecranon bursa, which were isointense to muscle. (B) T2weighted sagittal MRI; hyperintense to muscle, though less intense than fluid. (C) Following intravenous administration of gadolinium contrast, there was enhancement of the thin, smooth synovial membrane surrounding the olecranon bursa, but no enhancement of the soft tissue nodules.
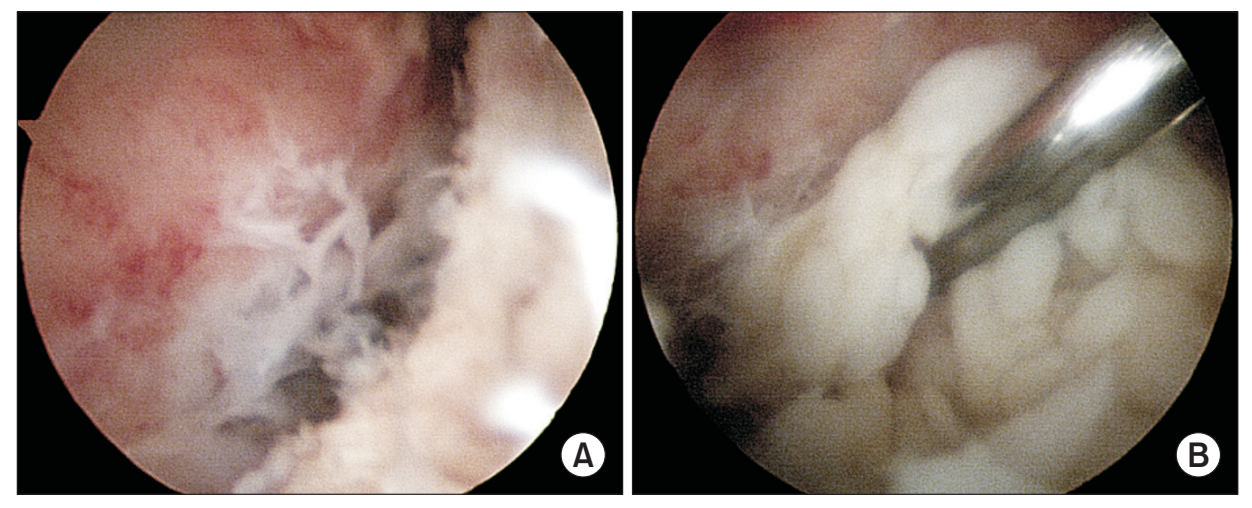

Fig. 4. (A) Inflammatory and proliferated synovial tissue in the olecranon bursa. (B) A mass of gray-white colored nodules in the bursa.

location of the bursa make it likely that a minor abrasion contaminated with soil or dirt may provide a portal for NTM entry. Alternatively, mycobacteria may be introduced by bursal aspiration or steroid injections into bursa. In our case, the patient had diabetes as the underlying disease and had received repeated injections; probably, diabetes or repeated injection histories or both were the sources of her NTM infection. Synovial chondromatosis may be associated with persistent olecranon bursitis; however, epidemiologically, it is unclear which one was first occurred between NTM infection and the synovial chondromatosis for the patient.

Rice body formation is most commonly observed in chronic rheumatoid arthritis, but may also occur in seronegative arthropathies and chronic low-grade synovial infections such as those that result from mycobacterial infection. ${ }^{5)}$ In addition, rice bodies resemble synovial chondromatosis both clinically and macroscopically. Macroscopically, they resemble polished white rice, leading to the term "rice bodies". Microscopically, rice bodies consist of an inner amorphous core of acidophilic material surrounded by collagen and fibrin. In synovial chondromatosis, grossly, multiple gray-white nodules are initially attached to the synovium, but may subsequently detach to form loose bodies. Microscopically, these nodules consist of hyaline cartilage with a mineralized chondroid matrix. In our case, multiple gray-white nodules were identified in the olecranon bursa, and histologically, they consisted of degenerated chondroid tissue.

Unmineralized synovial chondromatosis on MRI shows isointense or slightly hyperintense signals compared to the signal intensity of skeletal muscle in T1-weighted sequences and a hyperintense signal in T2-weighted sequences. In mineralized synovial chondromatosis, both T1- and T2-weighted sequences show hypointense signals. Other authors, however, have reported that T2-weighted images of synovial chondromatosis can show nodular filling defects within the hyperintense synovium, even when the nodules are not calcified. ${ }^{6)}$ The nodules are hyperintense to muscle, but less intense than fluid and synovium on T2-weighted images. ${ }^{7)}$ On MRI, rice bodies are also isointense on T1-weighted images and slightly hyperintense relative to muscle on T2-weighted images. ${ }^{8)}$ Rice bodies and synovial chondromatosis have similar characteristics when assessed visually or by MRI. Therefore, macroscopically and clinically, it is difficult to distinguish them. Thus, microscopic examination is 
needed to confirm the cause of rice body formation.

Recurrent Mycobacterium marinum tenosynovitis mimicking extra-articular synovial chondromatosis on MRI of the wrist has been reported, ${ }^{9)}$ and the authors suggested that rice bodies and uncalcified synovial chondromatosis may appear similar on MRI; thus, the possibility of an atypical mycobacterial infection should be considered. ${ }^{9)}$ Although, in our case, the preliminary diagnosis was secondary synovial chondromatosis associated with chronic olecranon bursitis, based on MRI, the patient had risk factors for infection such as diabetes mellitus and a history of repeated injections and recurrence. Before and during the operation, evaluation of the infectious condition could be done in various ways and such evaluation was important for early diagnosis and proper treatment without recurrence.

The specific antimycobacterial regimen depends upon the NTM species isolated and, for certain NTM species, depends on the results of in vitro susceptibility testing. It is important not to use antibacterial monotherapy since this can lead to the development of resistance. The duration of antimycobacterial therapy depends upon several factors, including the extent of disease, response to treatment, the causative species, and the immune status of the patient. ${ }^{1)}$ For most NTM species, antimycobacterial therapy should be continued for a minimum of six months but is sometimes provided for 12 months or longer. ${ }^{10)}$ In our case, the antimycobacterial regimen was determined in accordance with the basic principles of NTM treatment, with the regimen changed according to the results of the antimicrobial susceptibility test. With regard to the duration of medication, we recommend a minimum of 6 months. However, if there is a risk of infection such as that related to immunodeficiency or poor control of diabetes (as in our case), we recommended a medication duration of 6 months or longer, unless there are special situations such as side effects of the medication.

In conclusion, although synovial chondromatosis may occur in the olecranon bursa, to our knowledge, our case is the only one to have been reported. In addition, since uncalcified synovial chondromatosis and rice bodies may appear similar, both visually and on MRI, we suggest that clinicians should be aware of the possibility of other infections in cases of this type.

\section{References}

1. Garrigues GE, Aldridge JM 3rd, Toth AP, Stout JE. Nontuberculous mycobacterial olecranon bursitis: case reports and literature review. J Shoulder Elbow Surg. 2009;18(2):e1-5. doi: 10.1016/j.jse.2008.07.009.

2. Sim FH, Dahlin DC, Ivins JC. Extra-articular synovial chondromatosis. J Bone Joint Surg Am. 1977;59(4):492-5.

3. Yanagisawa H, Okada K, Sashi R. Posterior interosseous nerve palsy caused by synovial chondromatosis of the elbow joint. Clin Radiol. 2001;56(6):510-4. doi: 10.1053/crad.2001.0821.

4. Matsumoto T, Fujita K, Fujioka H, et al. Massive nonspecific olecranon bursitis with multiple rice bodies. J Shoulder Elbow Surg. 2004;13(6):680-3. doi: 10.1016/S1058274604001235.

5. Popert AJ, Scott DL, Wainwright AC, Walton KW, Williamson $\mathrm{N}$, Chapman JH. Frequency of occurrence, mode of development, and significance or rice bodies in rheumatoid joints. Ann Rheum Dis. 1982;41(2):109-17.

6. Rompen JC, Ham SJ, Molenaar WM, van Horn JR. Synovial chondromatosis of the wrist and hand--a case report. Acta Orthop Scand. 1999;70(6):627-9.

7. Coles MJ, Tara HH Jr. Synovial chondromatosis: a case study and brief review. Am J Orthop (Belle Mead NJ). 1997;26(1): 37-40.

8. Chen A, Wong LY, Sheu CY, Chen BF. Distinguishing multiple rice body formation in chronic subacromial-subdeltoid bursitis from synovial chondromatosis. Skeletal Radiol. 2002;31(2):119-21. doi: 10.1007/s002560100412.

9. Lee EY, Rubin DA, Brown DM. Recurrent Mycobacterium marinum tenosynovitis of the wrist mimicking extraarticular synovial chondromatosis on MR images. Skeletal Radiol. 2004;33(7):405-8. doi: 10.1007/s00256-004-0786-6.

10. Griffith DE, Aksamit T, Brown-Elliott BA, et al. An official ATS/ IDSA statement: diagnosis, treatment, and prevention of nontuberculous mycobacterial diseases. Am J Respir Crit Care Med. 2007;175(4):367-416. doi: 10.1164/rccm.200604571ST. 